International Journal of

Advanced Science and Convergence

\title{
Build a Secure Smart City by using Blockchain and Digital Twin
}

\author{
Youngjun Song ${ }^{1}$, Sunghyuck Hong ${ }^{2}$ \\ ${ }^{1}$ Department of Computer Engineering, Baekseok University, Cheonan, Republic of KOREA \\ ${ }^{2}$ Department of Smart IT, Baekseok University, Cheonan, Republic of KOREA
}

\begin{abstract}
Background/Objectives: As urbanization increases worldwide, so do the need for smart cities that enable more efficient city management. Based on smart city operational data, it explains the digital twin technologies required to predict outcomes and control according to circumstances through virtual simulations and presents related metaverse technologies. Methods/Statistical analysis: Introduction and explanation of smart city services using blockchain. The data infrastructure underlies each area, such as city operations, essential in a smart city. Findings: Therefore, blockchain is used to avoid hacking, data errors, and system failures of the existing centralized management and ensure data integrity and system stability by building a more secure server. In addition, blockchain enables the establishment of governance and free access to information through individual information sovereignty. Improvements/Applications: Most smart city models do not have a high level of authentication. Therefore, this study investigated a new smart city model that provides security and convenience to citizens by mutually complementing the virtual and real worlds by predicting urban situations through digital twins that can simulate impossible conditions in reality based on blockchain-based authentication.
\end{abstract}

\section{Index Terms}

Blockchain, City Service, Digital Twin, Smart City

\footnotetext{
Corresponding author : Sunghyuck Hong

shong@bu.ac.kr

- Manuscript received August 9, 2021

- Revised August 30, 2021 ; Accepted September 20, 2021

- Date of publication September 30, 2021

(c) The Academic Society of Convergence Science Inc.

2619-8150 () 2019 IJASC. Personal use is permitted, but republication/redistribution requires IJASC permission.
} 


\section{INTRODUCTION}

The digital twin, which originated in the manufacturing industry, plays an essential role in controlling various devices that the manufacturing industry values and rendering user jobs. Digital twins can help by simulating the real world in the virtual world and reflecting the results from optimized simulations in the real world [1-2]. With the development of IoT, CCTV, drones, intelligent transportation systems, and autonomous vehicles in building smart cities, data related to city management is increasing. At the same time, with the development of wireless communication networks such as artificial intelligence, cloud computing, the Internet, and $5 \mathrm{G}$, data processing and decision making are possible. These technological advances have made it possible to capture the structures and phenomena of the real world with the help of various sensors and quickly implement them in the virtual world through modeling. Land management with digital twins is a primary global concern. The OGC (Open Geospatial Consortium), an international spatial information standardization organization, is making significant efforts to standardize digital twin data [3-4]. As time goes by, the importance of information technologies such as IoT, AI, and significant data increases. The need will also increase as the application field increases in smart cities. In this paper, we explained digital twin and blockchain technologies and paid attention to establishing smart city data infrastructure with blockchain reliability and security advantages.

Chapter 2 describes the combination of blockchain and smart city, the digital twin, and the metaverse. Chapter 3 explains where blockchain is directly used in smart cities and the model that applies blockchain.

\section{RELATED WORK}

\section{A. Blockchain and Smart City}

According to a UN survey, cities with more than 10 million are expected to increase from 33 to 43 between 2018 and 2030. Urbanization increases economic power from the concentration of population and industry, but side effects such as traffic and environmental problems in cities occur in proportion. The occurrence of social issues related to urbanization and the increasing public budget reduction are the main reasons for increasing interest in building smart cities internationally. In a World Economic Forum survey, the smart city market, including the medical, security, and energy sectors, is expected to grow at an annual average of $23 \%$ between 2020 and 2024 to reach about $\$ 2.1$ trillion] [5]. The focus of smart cities is on efficient city management. In the case of blockchain, network participants can share data with a high degree of transparency and reliability without a central administrator. In the city's case, multiple stakeholders and data sharing among stakeholders are crucial for promoting city services. Therefore, blockchain can be used for such data exchange to build an efficient and secure system. For smart cities to improve service quality while solving social problems and operating efficiently, independent smart cities alone are not enough. Therefore, interoperability and coordination among smart city components are essential. For example, in the case of MaaS (Mobility as a Service), which connects multiple transportation systems in a smart city, blockchain can be used to exchange data and share profits among various carriers, such as settlement systems [5-6].

Linking many of the services and technologies that make up the smart city, using blockchain as a framework will create more value. Urban services can be automated through technology linkage. When automating urban services using blockchain networks, all interactions between users and providers are transparent and verifiable, and falsification of data is impossible no matter what. In energy management, blockchain can be used to make $\mathrm{P} 2 \mathrm{P}$ transactions more efficient by reducing transaction costs without intermediaries. A smart grid concept also increases energy efficiency by exchanging power consumption and generation in real-time between two directions of the existing power grid. Unlike existing nuclear and thermal power plants, renewable energy is affected by the environment, and installation locations vary. Therefore, the system cannot be used as a centralized system. In this case, energy transactions, such as trading surplus energy, will be carried out freely and securely through a microgrid system that uses the blockchain network, a distributed network. In smart cities, blockchain technology will track and manage urban data, enable frictionless transactions, and provide transparency in public services provided by the city. This way, reliability can be ensured and used as a cross-functional platform that connects different services [7].

\section{B. Digital Twin and Metaverse}

The big picture of digital twins and the metaverse implemented by the virtual world is similar, but they view the real world differently. Digital Twin is a technology that implements real-world objects such as cities, machines, and factories in the virtual world and works on that basis just as it does in the real world. The reality in the virtual world is called a digital twin because it embodies them like twins. In early digital twins, reality in the virtual world was copied, but with the recent development of IoT, 
computer, and artificial intelligence technologies, various scenarios that cannot be simulated due to realistic limitations can be implemented in the virtual world. Based on multiple sensors and actual data, it is possible to make realistic predictions and respond to problems in real-time. Predix, currently used by GE (General Electronics), is a cloud platform for integrating GE's equipment and software into the industrial internet to support smart factories. Predix securely collects and analyzes data from various machines and equipment in the real world. It is a digital twin platform that operates real-world equipment more efficiently through simulation based on the collected data [7-8]. Fig. 1 shows the structure of a digital twin that converts real objects into digital models based on model data and data collected by sensors in reality and uses them again in fact through data analysis, prediction, and visualization [8-9].

Fig. 1. Digital Twin Structure

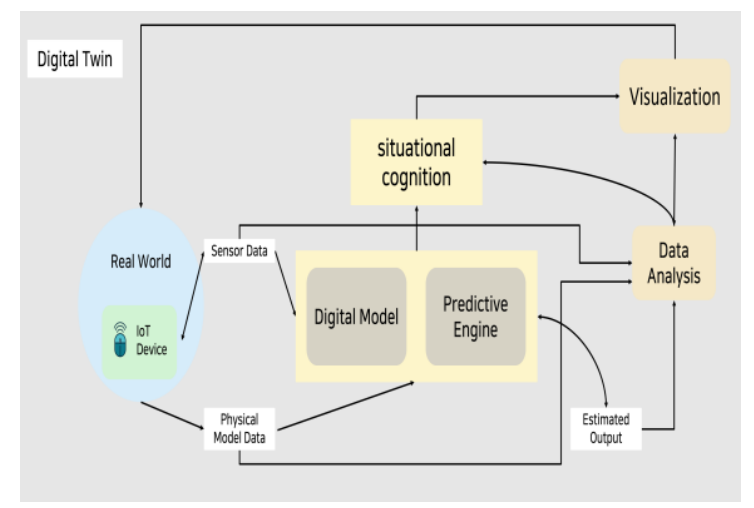

In metaverse, it is a term that combines Meta, which means transcendence, and Universe, which means the real world. In the metaverse, you experience different virtual worlds with avatars that you project yourself, but unlike digital twins, this does not mean that the virtual world has realized the real world. Metaverse is about creating and sharing different experiences and values through the interaction between the virtual world and the real world through the intersection with reality, away from the early 3D virtual world. In the case of BTS, the new song was released like a concert in the game Fortnite. Microsoft's remote collaboration platform Mesh is a metaverse platform based on MR (Mixed Reality). It allows users in different regions to feel like they are working in the same room through avatars. The metaverse is thus expanding to everyday areas such as games, industries, meetings, and shopping. In the future, the convergence of more and more domains of reality and activities with virtual ones will accelerate [10-11]. Metaverse consists of four elements represented by the virtual world, augmented reality, a mirror world implemented by adding additional information to the physical world, lifelogging that virtually reproduces information such as human emotions, movements, and bodies. Fig. 2 shows the concept of a metaverse consisting of four elements, combining reality and virtuality with general metaverse technologies such as XR (Extended Reality) and data technology.

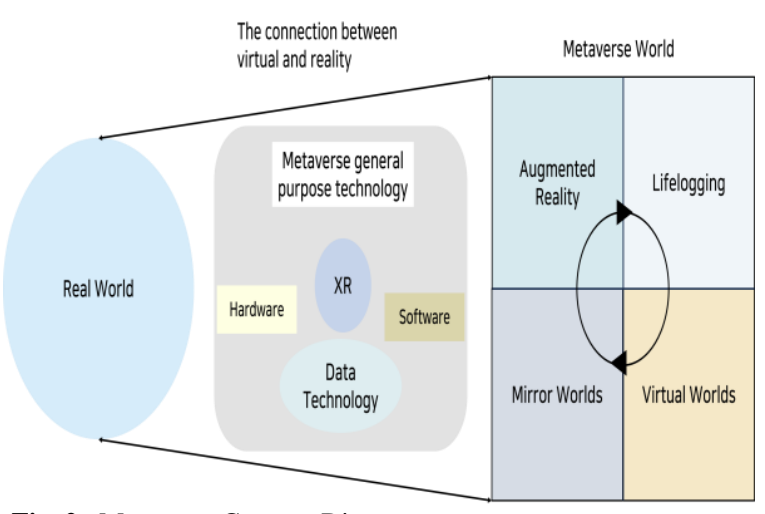

Fig. 2. Metaverse Concept Diagram

\section{The PROPOSED SMART City STANDARD MODEL}

Efforts to efficiently improve high energy consumption in smart cities are vital projects. When the urban energy blockchain network is formed, the energy information consumed in each city is recorded in the blockchain distribution ledger, and everyone can check the details. This has advantages in managing energy production and consumption as new and renewable energy such as solar energy is developed. In Korea, it is constrained by obtaining a certificate for a unique and renewable energy supply called REC and trading through the power exchange. In the case of energy blockchains, data integrity and stability are provided to users through the distributed ledger and hash technologies. This allows users to trade energy directly, without an intermediary. LO3 Energy has launched a blockchain-based microgrid platform in Brooklyn, USA, which builds a P2P energy market where local users generate, store, buy, and sell individual energy [12-13].

The development of cities and the increase of population naturally lead to improving citizens' quality of life and modern public administration. Improving administration is one of the most critical projects in smart cities, which provide more efficient administrative services to citizens. In the case of Smart Dubai, public documents are converted into blockchain-based electronic documents and based on this. A blockchain-based administrative system is established to provide online and mobile government services to citizens. Relying on the centralized 
system of existing institutions may cause problems such as hacking attacks or computer errors. To alleviate this problem, adopting blockchain technology can expand the scope of public service systems and create more secure and efficient channels for public service delivery. The level of privacy offered by blockchain is suitable for managing information data. The benefits of blockchain and smart contracts that provide system stability and data integrity in automating public administration, such as tax collection, property and land registration, benefit distribution, identity management, and government records control, can be maximized. Therefore, there is an advantage of increasing efficiency by securing trust in transaction records and reducing transaction time through the blockchain's distributed ledger. In addition, there is no need to have a central system, which is advantageous in terms of cost. Due to the nature of a smart city where all city data is gathered, the security and reliability of the blockchain will be a significant advantage. The efficient data infrastructure through the blockchain makes it possible to build a virtual city model through the digital twin with various data from cities such as terrain, transportation, and social problems. Accordingly, it is possible to intuitively analyze the urban situation through simulation of the virtual city model and respond quickly through more accurate decisions and control [14-15].

Fig. 3. Blockchain Smart City Mode

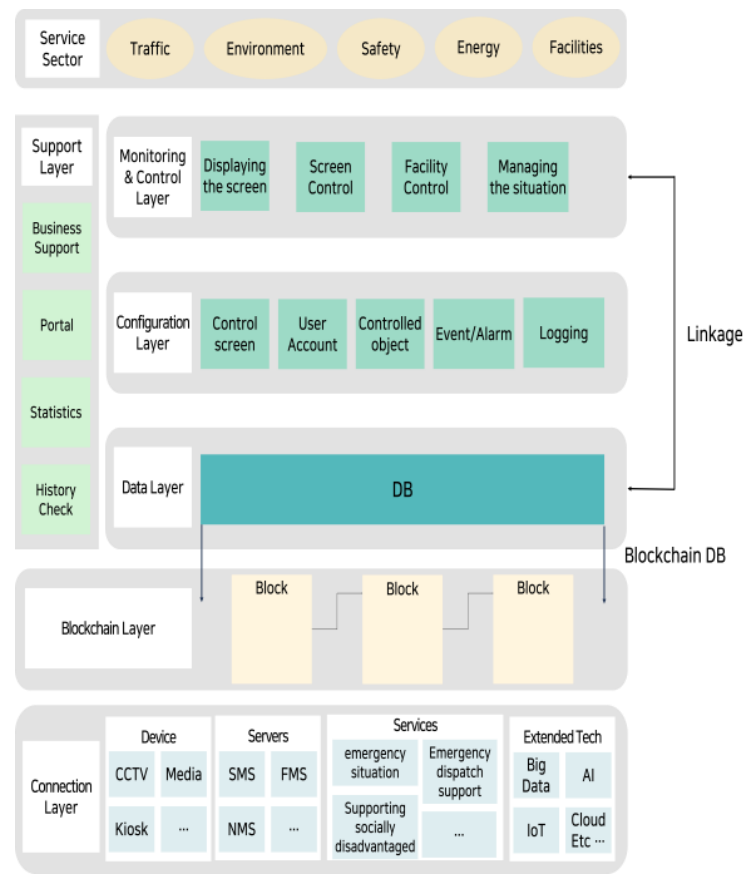

Smart cities have a broad base of practical applications and technologies, ranging from their overall infrastructure to data storage and processing to the service sectors that use them. Fig. 3 is a smart city model with blockchain. Each consists of a service sector, a support layer, a monitoring and control layer, a setting layer, a data layer, a blockchain layer, and a connection layer. In the service sector, transportation, urban environment, public safety, energy, and building management are provided by smart cities. The support layer includes statistics, portals, work support, and history queries used to support smart city operations. The monitoring and control layer corresponds to the control and management of cities, such as screen output, control, building control, and situation management. Due to the nature of smart cities operated based on all data in the city, it is crucial to increase the data integrity and system stability in the blockchain and DB partial composition. When a problem occurs in the centralized server, the blockchain, where each node is a single system, is more stable than the existing centralized DB, where the system fails.

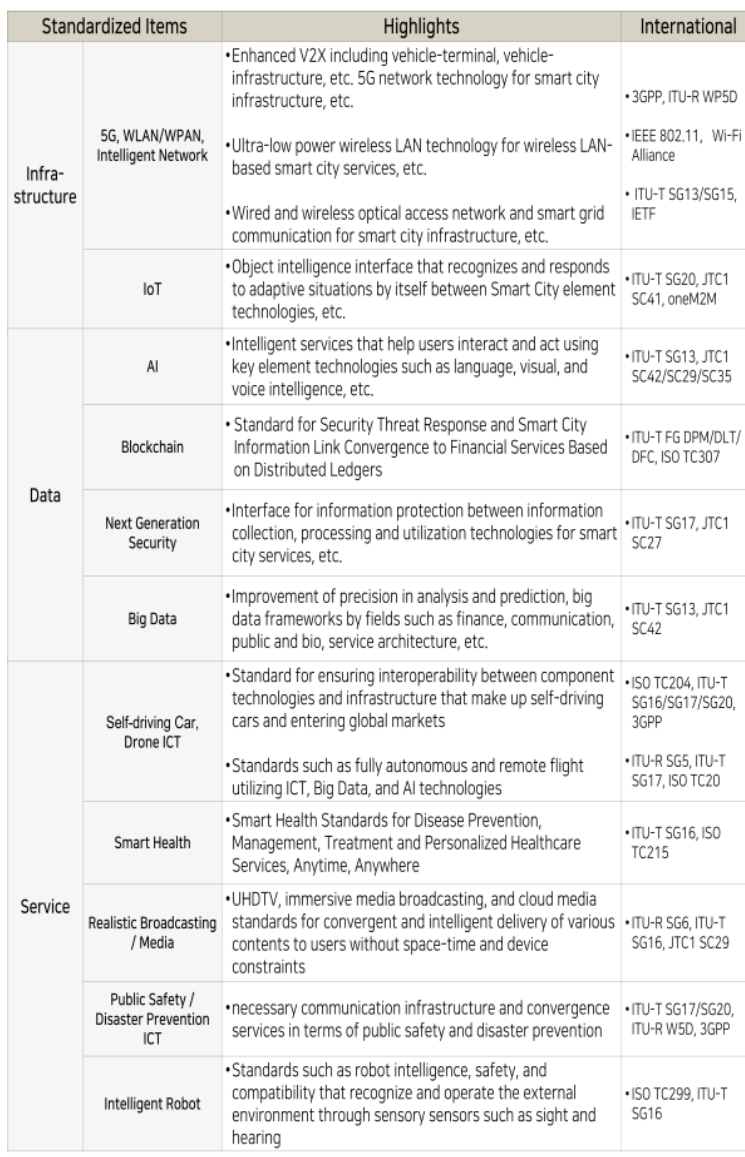

Fig. 4. Standardization of Smart City Element Technology at home and abroad

Moreover, blockchain provides high security and integrity of stored data by connecting cryptographybased hash technology and blocks into a chain. The connectivity layer corresponds to the devices, servers, services, and technologies associated with smart 
cities. Fig. 4 summarizes the technologies and content used in infrastructure, data, and services in Smart Cities and the international forums working on standardization.

\section{CONClusion}

The need for smart cities will inevitably arise in line with the global trend of growing urban population due to urbanization. Due to the nature of a smart city, where various technologies such as the IoT, AI, and Big Data are interconnected, the city's data infrastructure is essential. This study developed the model considering that blockchain technology, which can ensure data integrity and system stability, is suitable for managing data in smart cities. Since the recognition of information, sovereignty has been improved recently due to the nature of blockchain that allows access to data. It will be possible to develop a smart city in terms of governance and a system of self-sovereignty in which individuals directly manage information for identity verification. Most smart city models do not have a high proportion of authentication. Since most smart city models do not have a high percentage of authentication, this study can predict urban situations realistically through digital twins that can simulate impossible situations based on data within blockchain-based blockchain authentication. Based on situation prediction, it will be possible to provide efficient services to citizens in real-time through urban control.

\section{ACKNOWLEDGMENT}

This work was supported by the National Research Foundation of Korea(NRF) grant funded by the Korea government(MSIT) (No. 2020R1F1A1048684).

\section{REFERENCES}

[1] Ruohomaki, T., Airaksinen, E., Huuska, P., Kesaniemi, O., Martikka, M., \& Suomisto, J. (2018). Smart City Platform Enabling Digital Twin. 2018 International Conference on Intelligent Systems (IS).

[2] Maieron, M., \& Moreira de Oliveira, J. (2021). Open Data Integration in 3D CityGML-based Models Generation. Proceedings of the 23rd International Conference on Enterprise Information Systems.

[3] Son, H.-S. (2021). A Study on the Legislative Direction of Data Acts about Digital New Deal. Korean Constitutional Law Association, 27(1), 203-252.

[4] Kim, Y. H. (2020). Personal Information Protection Using Digital Twins in the Fourth Industrial Revolution. Journal of Digital Convergence, 18(6), 279-285.

[5] Written by Satoru Hori, P. F. (2021, April 6). How blockchain can empower smart cities. World Economic Forum. https://www.weforum.org/agenda/2021/04/howblockchain-can-empower-smart-cities-gtgs 21
[6] Mukherjee, P., Barik, R. K., \& Pradhan, C. (2020). A comprehensive proposal for blockchain-oriented smart city. Studies in Systems, Decision and Control, 55-87.

[7] Geißler, O. (2021, April 27). Blockchain und Smart Cities. Blockchain. https://www.blockchain-insider.de/blockchainund-smart-cities-a-1019295/.

[8] Tao, F., Zhang, M., \& Nee, A. Y. C. (2019). Background and concept of digital twin. Digital Twin Driven Smart Manufacturing, 3-28.

[9] KICT, A. (2018). Preliminary Study on KICT Digital Twin Technology. KICT, 134, 2018.

[10] Lee, B. K. (2021). The Metaverse World and Our Future. Review of Korea Contents Association, 19(1), 13-17.

[11] Suzuki, S.-nosuke, Kanematsu, H., Barry, D. M., Ogawa, N., Yajima, K., Nakahira, K. T., Shirai, T., Kawaguchi, M., Kobayashi, T., \& Yoshitake, M. (2020). Virtual experiments in metaverse and their applications to COLLABORATIVE projects: The framework and its significance. Procedia Computer Science, 176, 2125-2132.

[12] Cho, J. (2021). Implications and Roles of Blockchain for Smart City. Journal of the Korean Regional Science Association, 37(2), 35-48.

[13] Kim, K. B., Kim, G. C., \& Cho, H. J. (2018). Status and prospect of smart city in the fourth industrial revolution era. Journal of the Korea Convergence Society, 9(9), 191-197.

[14] Calzada, I. (2021). Devolving smart city citizenship: Smart city-regions, data devolution, and technological sovereignty. Smart City Citizenship, 141-177.

[15] Treiblmaier, H., Rejeb, A., \& Strebinger, A. (2020). Blockchain as a driver for smart city development: application fields and a comprehensive research agenda. Smart Cities, 3(3), 853-872. 